\title{
Flapwise bending vibration analysis of functionally graded rotating double-tapered beams
}

\author{
N. V. Ramesh Maganti ${ }^{1 *}$ and Mohan Rao Nalluri ${ }^{2}$
}

\begin{abstract}
Background: During the past decade, many researchers have focused on the vibration characteristics of stationary and rotating functionally graded beams employing various methods of analysis. Hitherto, the studies have been mainly confined to uniform beams. Hence, the present study is aimed at studying the free vibration characteristics of functionally graded double-tapered rotating cantilever beam with symmetric cross section.

Material and Method: This paper presents a method which considers the deformation variables to determine flapwise bending vibrations of rotating functionally graded doubletapered beam attached to a rigid hub. The tapered beam is characterized by continuously variable Young's modulus along the thickness direction according to a power law. The equations of motions are derived using hybrid deformation variables employing Lagrange's approach. Rayleigh-Ritz method is used to evaluate the frequencies of the beam.

Results: The effect of several parameters such as material composition gradient, taper ratios, hub radius ratio and rotational speed on flapwise bending natural frequencies of tapered beams are investigated.

Conclusions: Variation in taper ratios affects the frequency parameters of the beam. The frequency parameters are observed to increase with an increase in hub radius. The power law index has negligible influence on the trend of flapwise bending natural frequencies with an increase in angular speed for different taper ratios.
\end{abstract}

Keywords: Functionally graded beam; Rotating beam; Rayleigh-Ritz method; Flapwise vibration; Double-tapered beam

\section{Background}

Generally, to increase the strength as well as stability of the structure and to reduce the weight, tapered beams have practical importance at the design stage of structures, such as the helicopter blades, rotating machinery, wind turbine blades, etc. The design as well as analysis and estimation of dynamic characteristics of these structures is very difficult. Centrifugal inertia force due to rotation is one of the critical factors in evaluating the dynamic characteristics of the rotating structures. Many researchers have investigated the dynamic characteristics of the homogeneous rotating beams in the past. Tsai et al. (2011) investigated the free vibrations for inclined Euler beam rotating at constant angular speed using the co-rotational finite element method combined with floating frame method. In this work, the equations of motion were derived in terms of an inertial global coordinate system using

\footnotetext{
* Correspondence: ramesh.mnv@gmail.com

${ }^{1}$ Department of Mechanical Engineering, Nalla Malla Reddy Engineering

College, Hyderabad, Telangana 500088, India

Full list of author information is available at the end of the article
}

d'Alembert principle and principle of virtual work. A method based on the power series solution was proposed by Huang et al. (2010) for a slender beam rotating at high angular velocity, in which the beam was divided into equal segments for estimating flapwise vibration and coupled lagwise and axial vibration. New shape functions that include the rotating speed, element position, and centrifugal stiffening effect were considered to derive new finite element to evaluate free vibrations of beams rotating at high speeds by Gunda et al. (2009). Using $p$-version finite element method, the vibration characteristics of a rotating beam were obtained by Cheng et al. (2011). Fracture mechanics and Lagrange equation were used to obtain crack element stiffness matrix and $p$-version finite element model respectively in the analysis. The vibrations and reliability of rotating beams were studied considering the random properties under random excitations by Hosseini and Khadem (2007). Finite element method and space state analysis were applied to obtain the equations of motion. Free vibrations of tapered rotating beam in elastic

\section{勿 Springer}

(c) 2015 Maganti and Nalluri. Open Access This article is distributed under the terms of the Creative Commons Attribution 4.0 International License (http://creativecommons.org/licenses/by/4.0/), which permits unrestricted use, distribution, and reproduction in any medium, provided you give appropriate credit to the original author(s) and the source, provide a link to the Creative Commons license, and indicate if changes were made. 
and post elastic areas were evaluated using variational principles to derive the equations of motions in the works of Das et al. (2009). Assuming the centrifugal force as constant, the differential equation of motion of rotating beam was reduced as stiff string and natural frequencies of uniform and tapered beams were evaluated by Ganesh and Ganguli (2013) using Rayleigh-Ritz method. A new finite element was developed by Babu Gunda and Ganguli (2008) satisfying the governing equation of Euler-Bernoulli rotating beams to study the static and dynamic behavior of rotating beam. Lin et al. (2006) evaluated bending-bending vibrations of rotating damped beam with pre-twist and an elastically restrained root and their effects on the complex frequencies of rotating beams were observed. Banerjee and Jackson (2013) studied on free vibration characteristics of a rotating tapered Rayleigh beam considering centrifugal stiffening, outboard force, arbitrary hub radius, and rotary inertia.

Smooth and continuous variation in material properties in one or more directions in case of structures like beams plates and shells, which are made from functionally graded materials (FGMs), exhibits several advantages over homogeneous and laminated composite materials. During the past one decade, many researchers have turned their interest to study the vibration and dynamic characteristics of FGM structures. Sankar (2001) developed a solution for a functionally graded beam subjected to transverse loads, whose Young's modulus was assumed to vary in thickness direction, and found that the stress concentration depends on the distribution of constituent material of the beam. Based on two-dimensional theory of elasticity, Ying et al. (2008) proposed an exact solution for free vibrations of functionally graded beams resting on elastic foundation wherein material properties were assumed to vary in thickness direction as per exponential law. Higher order shear deformation theory was implemented by Kadoli et al. (2008) to study the behavior of the FG beam with variation of material composition based on power law under uniformly distributed load with different end conditions. Differential equation and its solution for deflection of FGM beams resting on elastic foundation using second-order theory were obtained by Murin et al. (2013) and used in free vibration analysis of the beams. Considering the fourth-order differential equation of the secondorder beam theory, modal analysis was carried out for beams by Aminbaghai et al. (2012). Using the first-order shear deformation theory, dynamic stiffness matrix was derived by Ziane et al. (2013) to evaluate the free vibrations of functionally graded box beam.

The above studies chiefly focused on the evaluation of dynamic characteristics of homogeneous stationary and rotating uniforms and FGM beams that are stationary. For the design and analysis of tapered rotating structures like wind turbine blades, helicopter blades, rotating machinery, etc., vibration characteristics are vital parameters. The knowledge on these characteristics play a very important role in design stage for studying the dynamic behavior and to avoid structural resonance. The flapwise and chordwise dynamic behavior of the beam depends on the nature of the transverse loads, geometry of the component, and the boundary conditions. Hitherto, the studies have been mainly confined to uniform beams. Hence, the present study is aimed at studying the free vibration characteristics of double-tapered rotating cantilever beam with symmetric cross section. The beam material considered is FGM and the variables considered are taper ratio, composition variation of material as per power law index, hub radius ratio, and rotational speed. The results so obtained are compared with those reported in the earlier works based on different approaches.

\section{Material and Method}

\section{Functionally graded beam}

Consider a doubly symmetric cross-section functionally graded beam with length, $L$, width, $w_{0}$, and height, $h_{0}$, at the root of the beam and width, $w$, and height, $h$, at the tip of the beam as shown in Fig. 1. The graded material properties vary symmetrically along thickness direction from the core towards the surface according to a power law:

$$
P_{(z)}=P_{(m)}+\left(P_{(c)}-P_{(m)}\right)\left|\frac{2 \times z}{h}\right|^{n}
$$

where $P_{(z)}$ represents an effective material property (i.e., density, $P_{(z)}$, or Young's modulus, $\left.E_{(z)}\right)$ and $P_{(m)}$ and $P_{(c)}$ are metallic and ceramic properties, respectively.

The volume fraction exponent or power law index, $n$, is a variable whose value is greater than or equal to zero and the variation in properties of the beam depends on its magnitude. Structure is constructed with functionally graded material with ceramic rich at top and bottom surfaces (at $z$ $=h / 2$ and $-h / 2$ ) with protecting metallic core $($ at $z=0)$.

\section{Equations of motion}

For the beam considered in this study, the equations of motion are obtained with the following assumptions:

1. There is a smooth and continuous variation in properties along thickness direction.

2. The neutral and centroidal axes in the cross section of the rotating beam coincide so that effects due to eccentricity and torsion can be negligible.

3. Cross section of the beam varies uniformly along its length with the uniform taper.

4. Shear and rotary inertia effects of the beam are neglected due to slender shape of the beam.

Figure 1 shows the deformation of the neutral axis of a beam fixed to a rigid hub rotating about the axis $z$. The tapered beam is attached to a rigid hub which rotates with 


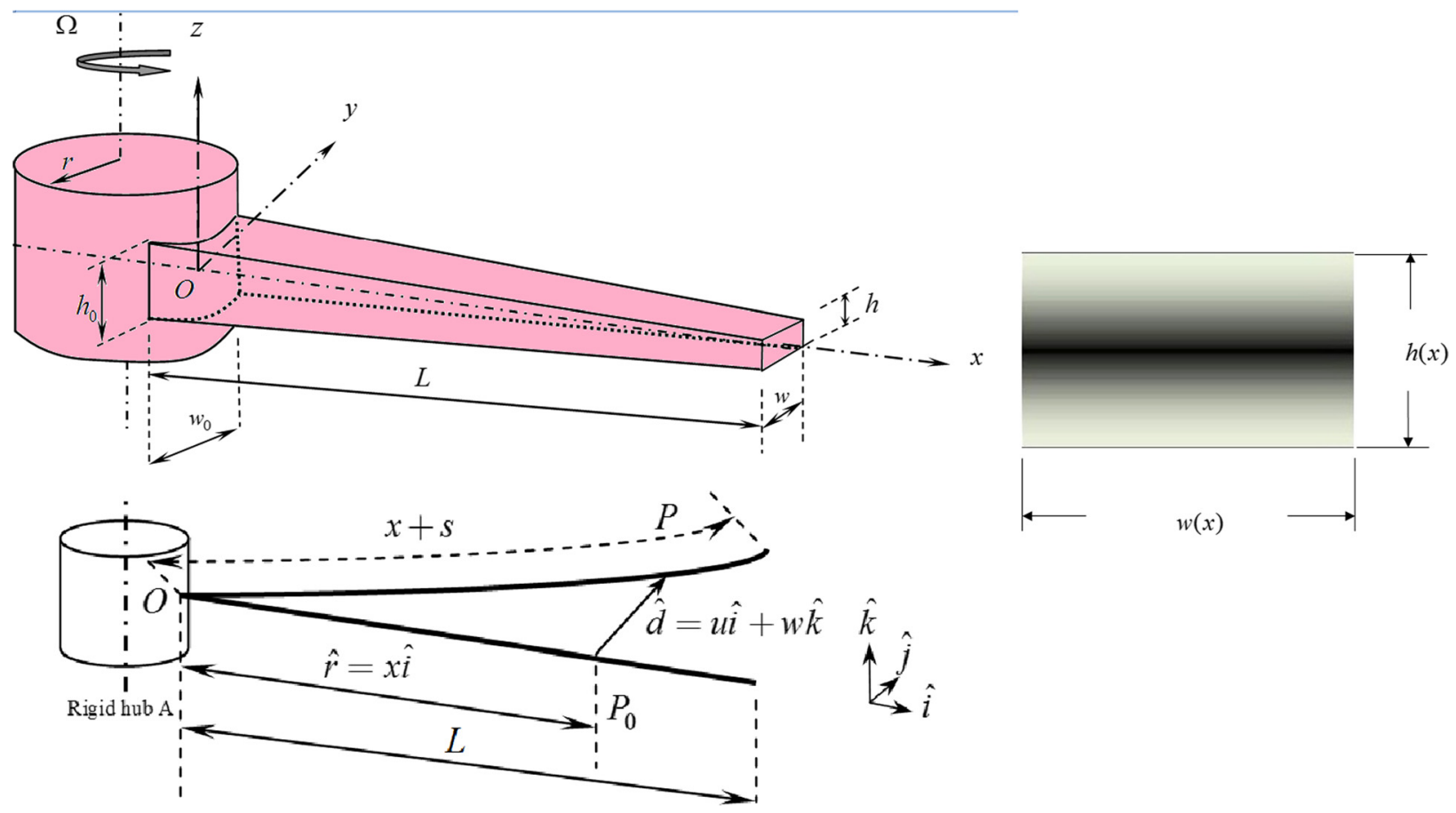

Fig. 1 Configuration of the functionally graded double-tapered rotating beam

constant angular speed and no external force acts on the beam. The beam rotates at an angular speed of $\Omega(t)$ about the $z$-axis. In the present work, a hybrid set of Cartesian variable $w$ and a non-Cartesian variable $s$ is approximated by spatial functions and corresponding coordinates are employed to derive the equations of motion. The position of a generic point on the neutral axis of the doubletapered beam before deformation located at $P_{0}$ changes to $P$ after deformation and its elastic deformation is denoted as $\hat{d}$ that has two components in two-dimensional space.

\section{Approximation of deformation variables}

Using the Rayleigh-Ritz assumed mode method, the deformation variables are approximated as

$$
s(x, t)=\sum_{j=1}^{\mu_{1}} \phi_{1 j}(x) q_{1 j}(t)
$$

and

$$
w(x, t)=\sum_{j=1}^{\mu_{3}} \phi_{3 j}(x) q_{3 j}(t)
$$

In the above equations, $\phi_{1 j}$ and $\phi_{3 j}$ are the assumed modal functions (test functions) for $s$ and $w$, respectively. Any compact set of functions which satisfies the essential boundary conditions of the cantilever beam can be used as the test functions. The $q_{i j} \mathrm{~s}$ are the generalized coordinates and $\mu_{1}$ and $\mu_{3}$ are the number of assumed modes used for $s$ and $w$, respectively. The total number of modes, $\mu$, is equal to the sum of individual modes, i.e., $\mu=\mu_{1}+\mu_{3}$.
The geometric relation between the arc length stretch $s$ and Cartesian variables $u$ and $w$ presented by Yoo and Shin (1998) as

$$
s=u+\frac{1}{2} \int_{0}^{x}\left[\left(w^{\prime}\right)^{2}\right] d \sigma
$$

where a symbol with a prime (') represents the partial derivative of the symbol with respect to the integral domain variable.

\section{Strain energy of the system}

The total elastic strain energy of the beam considering the assumptions given in "Functionally graded beam" section can be written as

$$
E_{s}=\frac{1}{2} E(z) A(x) \int_{L}\left(\frac{d s}{d x}\right)^{2} d x+\frac{1}{2} E(z) I_{y}(x) \int_{L}\left(\frac{d^{2} w}{d x^{2}}\right)^{2} d x(\mathrm{i}=1,2, \mu)
$$

where $I_{y(x)}$ is the second moment of area of the tapered beam.

For the beam tapers in two planes, the general parameters of the beam are given by

$$
\begin{aligned}
& h(x)=h_{o}\left(1-\tau_{h} \frac{x}{L}\right) \\
& w(x)=w_{o}\left(1-\tau_{w} \frac{x}{L}\right)
\end{aligned}
$$




$$
\begin{aligned}
& A(x)=A_{0}\left(1-\tau_{w} \frac{x}{L}\right)\left(1-\tau_{h} \frac{x}{L}\right) \\
& I_{y}(x)=I_{0 y}\left(1-\tau_{w} \frac{x}{L}\right)\left(1-\tau_{h} \frac{x}{L}\right)^{3}
\end{aligned}
$$

where $\tau_{w}$ and $\tau_{h}$ are the width taper ratio and height taper ratio respectively and are defined as

$$
\begin{aligned}
\tau_{w} & =\left(1-\frac{w}{w_{0}}\right) \\
\tau_{h} & =\left(1-\frac{h}{h_{0}}\right)
\end{aligned}
$$

where $A_{0}$ and $I_{0 y}$ are area of cross section and area moment of inertia of the beam at the root of the tapered beam respectively and are defined as

$$
\begin{aligned}
& A_{0}=w_{0} h_{0} \\
& I_{0 y}=\frac{b_{0} w_{0}^{3}}{12}
\end{aligned}
$$

\section{Kinetic energy of the system}

The velocity of a generic point $P$ can be obtained as

$$
\vec{v}^{P}=\vec{v}^{O}+\frac{{ }^{A} d \vec{p}}{d t}+\vec{\omega}^{A} \times \vec{p}
$$

where $\vec{v} O$ is the velocity of point $O$ that is a reference point identifying a point fixed in the rigid frame $A ; \vec{\omega} O$ vector $\vec{P}$ in the reference frame $A$ and the terms $\vec{P}, \vec{v} o$, and $\vec{\omega} A$ can be expressed as follows

$$
\begin{aligned}
& \vec{p}=(x+u) \hat{i}+v \hat{j} \\
& \vec{v}^{O}=r \Omega \hat{j} ; \\
& \vec{\omega}^{A}=\Omega \hat{k} \\
& \vec{v}^{p}=(\dot{u}-\Omega v) \hat{i}+[\dot{v}+\Omega(r+x+u)] \hat{j}
\end{aligned}
$$

where $\hat{\imath}, \hat{\jmath}$, and $\hat{k}$ are orthogonal unit vectors fixed in $A, r$ is the distance from the axis of rotation to point $O$ (i.e., radius of the rigid frame), and $\Omega$ is the angular speed of the rigid

\begin{tabular}{|c|c|c|c|c|c|}
\hline$\tau_{h}$ & $\begin{array}{l}T_{b} \\
0.0\end{array}$ & 0.2 & 0.4 & 0.6 & 0.8 \\
\hline & \multicolumn{5}{|c|}{ (a) Fundamental frequency } \\
\hline \multirow[t]{2}{*}{0.0} & 3.15601 & 3.76285 & 4.09697 & 4.58531 & 5.39758 \\
\hline & $3.51602^{\mathrm{a}}$ & $3.76286^{a}$ & $4.09698^{\mathrm{a}}$ & $4.58531^{\mathrm{a}}$ & $5.39759^{\mathrm{a}}$ \\
\hline \multirow[t]{2}{*}{0.2} & 3.60827 & 3.85511 & 4.18932 & 4.67815 & 5.49265 \\
\hline & $3.60828^{a}$ & $3.85512^{\mathrm{a}}$ & $4.18932^{\mathrm{a}}$ & $4.67816^{\mathrm{a}}$ & $5.49266^{\mathrm{a}}$ \\
\hline \multirow[t]{2}{*}{0.4} & 3.73707 & 3.98419 & 4.31877 & 4.80842 & 5.62551 \\
\hline & $3.73708^{a}$ & $3.98419^{a}$ & $4.31878^{a}$ & $4.80842^{a}$ & $5.62552^{\mathrm{a}}$ \\
\hline \multirow[t]{2}{*}{0.6} & 3.93427 & 4.18234 & 4.51799 & 5.00903 & 5.82882 \\
\hline & $3.93428^{\mathrm{a}}$ & $4.18235^{a}$ & $4.51799^{a}$ & $5.00903^{\mathrm{a}}$ & $5.82882^{\mathrm{a}}$ \\
\hline \multirow[t]{2}{*}{0.8} & 4.29250 & 4.54369 & 4.88244 & 5.37615 & 6.19641 \\
\hline & $4.29249^{a}$ & $4.54368^{a}$ & $4.88244^{a}$ & $5.37614^{a}$ & $6.19639^{\mathrm{a}}$ \\
\hline
\end{tabular}
frame. Using Eq. 14, the kinetic energy of the rotating beam is derived as

$$
E_{k}=\frac{1}{2} \int_{0}^{L} \rho(z) A(x) \vec{v}^{p} \cdot \vec{v}^{p} d x
$$

In which, $A_{(x)}$ is the area of cross section of the tapered beam, $P_{(z)}$ is the mass density, and $L$ is the length of the beam.
Table 1 Comparison of the non-dimensional lowest three flapwise bending natural frequencies of a homogenous stationary beam with different taper ratios $(\delta=0)$

(b) Second natural frequency

\begin{tabular}{llllll}
0.0 & 22.0344 & 22.5018 & 23.1186 & 24.0210 & 25.6558 \\
& $22.0345^{\mathrm{a}}$ & $22.5018^{\mathrm{a}}$ & $23.1186^{\mathrm{a}}$ & $24.0211^{\mathrm{a}}$ & $25.6558^{\mathrm{a}}$ \\
0.2 & 20.6210 & 21.0567 & 21.6327 & 22.4774 & 24.0153 \\
& $20.6210^{\mathrm{a}}$ & $21.0567^{\mathrm{a}}$ & $21.6327^{\mathrm{a}}$ & $22.4774^{\mathrm{a}}$ & $24.0153^{\mathrm{a}}$ \\
\multirow{2}{*}{0.4} & 19.1138 & 19.5166 & 20.0500 & 20.8343 & 22.2710 \\
& $19.1138^{\mathrm{a}}$ & $19.5166^{\mathrm{a}}$ & $38.4920^{\mathrm{a}, \mathrm{b}}$ & $20.8343^{\mathrm{a}}$ & $22.2710^{\mathrm{a}}$ \\
\multirow{2}{*}{0.6} & 17.4879 & 17.8557 & 18.3441 & 19.0649 & 20.3953 \\
& $17.4879^{\mathrm{a}}$ & $17.8557^{\mathrm{a}}$ & $18.3441^{\mathrm{a}}$ & $19.0649^{\mathrm{a}}$ & $20.3952^{\mathrm{a}}$ \\
\multirow{2}{*}{0.8} & 15.7473 & 16.0731 & 16.5132 & 17.1668 & 18.3871 \\
& $15.7427^{\mathrm{a}}$ & $16.0725^{\mathrm{a}}$ & $16.5123^{\mathrm{a}}$ & $17.1657^{\mathrm{a}}$ & $18.3855^{\mathrm{a}}$
\end{tabular}

(c) Third natural frequency

$\begin{array}{llllll}0.0 & 61.6972 & 62.1525 & 62.7763 & 63.7515 & 65.7470 \\ & 61.6972^{\mathrm{a}} & 62.1525^{\mathrm{a}} & 62.7763^{\mathrm{a}} & 63.7515^{\mathrm{a}} & 65.7470^{\mathrm{a}} \\ 0.2 & 56.1923 & 56.6304 & 57.2258 & 58.1467 & 60.0096 \\ & 56.1923^{\mathrm{a}} & 56.6303^{\mathrm{a}} & 57.2257^{\mathrm{a}} & 58.1466^{\mathrm{a}} & 60.0094^{\mathrm{a}} \\ 0.4 & 50.3537 & 50.7715 & 51.3348 & 52.1965 & 53.9177 \\ & 50.3537^{\mathrm{a}} & 50.7714^{\mathrm{a}} & 51.3346^{\mathrm{a}} & 52.1963^{\mathrm{a}} & 53.9173^{\mathrm{a}} \\ 0.6 & 44.0252 & 44.4180 & 44.9437 & 45.7391 & 47.3066 \\ & 44.0248^{\mathrm{a}} & 44.4175^{\mathrm{a}} & 44.9432^{\mathrm{a}} & 45.7384^{\mathrm{a}} & 47.3051^{\mathrm{a}} \\ 0.8 & 36.8955 & 37.2558 & 37.7358 & 38.4555 & 39.8568 \\ & 36.8846^{\mathrm{a}} & 37.2439^{\mathrm{a}} & 37.7223^{\mathrm{a}} & 38.4392^{\mathrm{a}} & 39.8336^{\mathrm{a}}\end{array}$

${ }^{a}$ Results of Ozgumus and Kaya (2006)

${ }^{\text {DT}}$ The trends in the second frequency reported by Ozgumus and Kaya (2006) should exhibit decreasing trend with an increase in taper ratio. However, at $\tau_{b}=\tau_{h}=0.4$, a higher value is reported. It is opened that it is a typographical error

\section{Equation of motion}

Substituting Eqs. 2 and 3 into Eqs. 5 and 19 and taking partial derivatives of $E_{k}$ and $E_{s}$ with respect to $q_{i j}$ and $q_{i j}$, neglecting the higher order non-linear terms, the equation of motion for the tapered beam is formulated using the 
Lagrange's method. The Lagrange's equation of motion for free vibration of distributed parameter system can be written as

$$
\frac{d}{d t}\left(\frac{\partial E_{k}}{\partial \dot{q} i}\right)-\frac{\partial E_{k}}{\partial q i}+\frac{\partial E_{s}}{\partial q i}=0 \mathrm{i}=1,2,3 \mu
$$

The linearized equations of motion can be obtained as follows

$$
\begin{aligned}
& \sum_{j=1}^{\mu_{1}}\left[\left(\rho_{(z)} \int_{0}^{L} A_{0}\left(1-\tau_{w} \frac{x}{L}\right)\left(1-\tau_{h} \frac{x}{L}\right) \phi_{1 i} \phi_{1 j} d x\right)\right. \\
& \ddot{q}_{1 j}-2 \Omega\left(\rho_{(z)} \int_{0}^{L} A_{0}\left(1-\tau_{w} \frac{x}{L}\right)\left(1-\tau_{h} \frac{x}{L}\right) \phi_{1 i} \phi_{2 j} d x\right) \dot{q}_{i j} \\
& -\Omega^{2}\left(\rho_{(z)} \int_{0}^{L} A_{0}\left(1-\tau_{w} \frac{x}{L}\right)\left(1-\tau_{h} \frac{x}{L}\right) \phi_{1 i} \phi_{1 j} d x\right) q_{1 j} \\
& +\left(E_{(z)} \int_{0}^{L} I_{0 z}\left(1-\tau_{b} \frac{x}{L}\right)^{3}\left(1-\tau_{h} \frac{x}{L}\right) \phi_{1 i}^{\prime} \phi_{1 j}^{\prime} d x\right) q_{1 j} \\
& =\Omega^{2} \rho_{(z)} \int_{0}^{L} A_{0}\left(1-\tau_{w} \frac{x}{L}\right)\left(1-\tau_{h} \frac{x}{L}\right) x \phi_{1 i} d x \\
& +\Omega^{2} \rho(z) \int_{0}^{L} A_{0}\left(1-\tau_{w} \frac{x}{L}\right)\left(1-\tau_{h} \frac{x}{L}\right) \phi_{1 i} d x
\end{aligned}
$$

$$
\begin{aligned}
& \sum_{j=1}^{\mu_{3}}\left[\left(\rho_{(z)} \int_{0}^{L} A_{0}\left(1-\tau_{w} \frac{x}{L}\right)\left(1-\tau_{h} \frac{x}{L}\right) \phi_{3 i} \phi_{3 j} d x\right) \ddot{q}_{3 j}\right. \\
+ & \left(E_{(z)} I_{0 z} \int_{0}^{L}\left(1-\tau_{b} \frac{x}{L}\right)\left(1-\tau_{h} \frac{x}{L}\right)^{3} \phi_{3 i}^{\prime \prime} \phi_{3 j}^{\prime \prime} d x\right) q_{3 j} \\
+ & \Omega^{2}\left\{r\left(\rho_{(z)} \int_{0}^{L} A_{0}\left(1-\tau_{w} \frac{x}{L}\right)\left(1-\tau_{h} \frac{x}{L}\right)(L-x) \phi_{3 i}^{\prime} \phi_{3 j}^{\prime} d x\right) q_{3 j}\right. \\
+ & \left.\left.\left(\rho_{(z)} \int_{0}^{L} A_{0}\left(1-\tau_{w} \frac{x}{L}\right)\left(1-\tau_{h} \frac{x}{L}\right) \frac{1}{2}\left(L^{2}-x^{2}\right) \phi_{3 i}^{\prime} \phi_{3 j}^{\prime} d x\right) q_{3 j}\right\}\right]=0
\end{aligned}
$$

where a symbol with double prime (") represents the second derivative of the symbol with respect to the integral domain variable.

\begin{tabular}{|c|c|c|c|c|c|c|c|}
\hline \multirow[t]{2}{*}{$\bar{\delta}$} & \multirow[t]{2}{*}{ Y } & \multicolumn{2}{|c|}{ First frequency } & \multicolumn{2}{|c|}{ Second frequency } & \multicolumn{2}{|c|}{ Third frequency } \\
\hline & & $\begin{array}{l}\text { Present } \\
\text { approach }\end{array}$ & $\begin{array}{l}\text { Ref. (Ozgumus and Kaya } \\
\text { 2006) }\end{array}$ & $\begin{array}{l}\text { Present } \\
\text { approach }\end{array}$ & $\begin{array}{l}\text { Ref. (Ozgumus and Kaya } \\
\text { 2006) }\end{array}$ & $\begin{array}{l}\text { Present } \\
\text { approach }\end{array}$ & $\begin{array}{l}\text { Ref. (Ozgumus and Kaya } \\
\text { 2006) }\end{array}$ \\
\hline \multirow[t]{4}{*}{0.0} & 1 & 4.017 & 3.986 & 18.501 & 18.474 & 47.448 & 47.417 \\
\hline & 2 & 4.547 & 4.436 & 19.045 & 18.936 & 47.995 & 47.871 \\
\hline & 3 & 5.308 & 5.092 & 19.917 & 19.683 & 48.891 & 48.619 \\
\hline & 4 & 6.211 & 5.878 & 21.077 & 20.685 & 50.117 & 49.645 \\
\hline \multirow[t]{4}{*}{2.0} & 1 & 4.482 & 4.386 & 18.974 & 18.879 & 47.939 & 47.830 \\
\hline & 2 & 6.033 & 5.742 & 20.819 & 20.473 & 49.902 & 49.486 \\
\hline & 3 & 7.955 & 7.452 & 23.562 & 22.879 & 52.995 & 52.120 \\
\hline & 4 & 10.027 & 9.310 & 26.918 & 25.866 & 57.013 & 55.581 \\
\hline \multirow[t]{4}{*}{0.5} & 1 & 4.138 & 4.090 & 18.621 & 18.576 & 47.571 & 47.521 \\
\hline & 2 & 4.961 & 4.797 & 19.504 & 19.332 & 48.480 & 48.281 \\
\hline & 3 & 6.082 & 5.777 & 20.892 & 20.531 & 49.953 & 49.520 \\
\hline & 4 & 7.359 & 6.905 & 22.689 & 22.099 & 51.940 & 51.201 \\
\hline \multirow[t]{4}{*}{1.0} & 1 & 4.256 & 4.191 & 18.739 & 18.677 & 47.694 & 47.624 \\
\hline & 2 & 5.343 & 5.132 & 19.953 & 19.720 & 48.959 & 48.686 \\
\hline & 3 & 6.766 & 6.386 & 21.821 & 21.343 & 50.990 & 50.403 \\
\hline & 4 & 8.346 & 7.793 & 24.187 & 23.425 & 53.692 & 52.706 \\
\hline
\end{tabular}

\section{Dimensionless transformation}

For the analysis, Eqs. 21 and 22 may be obtained in dimensionless form by introducing following dimensionless variables in to the equations:

$$
\begin{gathered}
\tau=\frac{t}{T} \\
\xi=\frac{x}{L} \\
\theta_{j}=\frac{q_{j}}{L}
\end{gathered}
$$

Table 2 Variation of the first three non-dimensional natural frequencies, with respect to the rotational speed parameter, $\gamma$, and the hub radius parameter, $\delta\left(\tau_{b}=0, \tau_{h}=0.5\right)$ 


$$
\begin{aligned}
& \delta=\frac{r}{L} \\
& \gamma=T \Omega
\end{aligned}
$$

where $\tau, \delta$, and $\gamma$ refer to dimensionless time, hub radius ratio, and dimensionless angular speed, respectively.

\section{Analysis of flapwise bending natural frequencies}

Equation 22 governs the flapwise bending vibration of the double-tapered rotating beam which is not coupled with Eq. 21. With the assumption that the first stretching natural frequency of an Euler beam far separated from the first natural frequency, Eq. 21 is ignored in present analysis. Equation 22 involves the parameters $L$, $\Omega, x, E$, and $P$, which are the properties of the beam.

After introducing the dimensionless variable from Eqs. 23, 24, 25, 26, and 27 in Eq. 22, the equation results

$$
\begin{aligned}
& \sum_{j=i}^{\mu_{3}}\left[\left(\int_{0}^{1}\left(1-\tau_{w} \xi\right)\left(1-\tau_{h} \xi\right) \psi_{3 i} \psi_{3 j} d \zeta\right) \ddot{\theta}_{2 j}\right. \\
& +\left(\int_{0}^{1}\left(1-\tau_{b} \xi\right)\left(1-\tau_{h} \xi\right)^{3} \psi_{3 i}^{\prime \prime} \psi_{3 j}^{\prime \prime} d \zeta\right) \theta_{3 j} \\
& +\gamma^{2}\left\{\left(\int_{0}^{1} \delta\left(1-\tau_{w} \xi\right)\left(1-\tau_{h} \xi\right)(1-\xi) \psi_{3 i}^{\prime} \psi_{3 j}^{\prime} d \zeta\right) \theta_{3 j}\right. \\
& \left.\left.\left.+\left(\frac{1}{2} \int_{0}^{1}\left(1-\tau_{w} \xi\right)\left(1-\tau_{h} \xi\right)\left(1-\xi^{2}\right) \psi_{3 i}^{\prime} \psi_{3 j}^{\prime} d \zeta\right)\right) \theta_{3 j}\right\}\right]=0
\end{aligned}
$$

where

$$
T=\left(\frac{\rho A_{0} L^{4}}{E_{(z)} I_{0 y}}\right)^{\frac{1}{2}}
$$

Equation 28 can be written as

$$
\sum_{j=1}^{\mu_{3}}\left[M_{i j}^{33} \ddot{\theta}_{3 j}+K_{i j}^{B 3} \theta_{3 j}+\gamma^{2}\left(K_{i j}^{G 3}-M_{i j}^{33}\right) \theta_{3 j}\right]=0
$$

where

$$
M_{i j}^{a b}=\int_{0}^{1}\left(1-\tau_{w} \xi\right)\left(1-\tau_{h} \xi\right) \psi_{a i} \psi_{b j} d \xi
$$

Table 3 Properties of metallic (steel) and ceramic (alumina) materials

\begin{tabular}{lcc}
\hline Properties of materials & Steel & Alumina $\left(\mathrm{Al}_{2} \mathrm{O}_{3}\right)$ \\
\hline Young's modulus $\mathrm{E}(\mathrm{GPa})$ & 214.00 & 390.00 \\
Material density $\rho\left(\mathrm{kg} / \mathrm{m}^{3}\right)$ & 7800.00 & 3200.00 \\
\hline
\end{tabular}

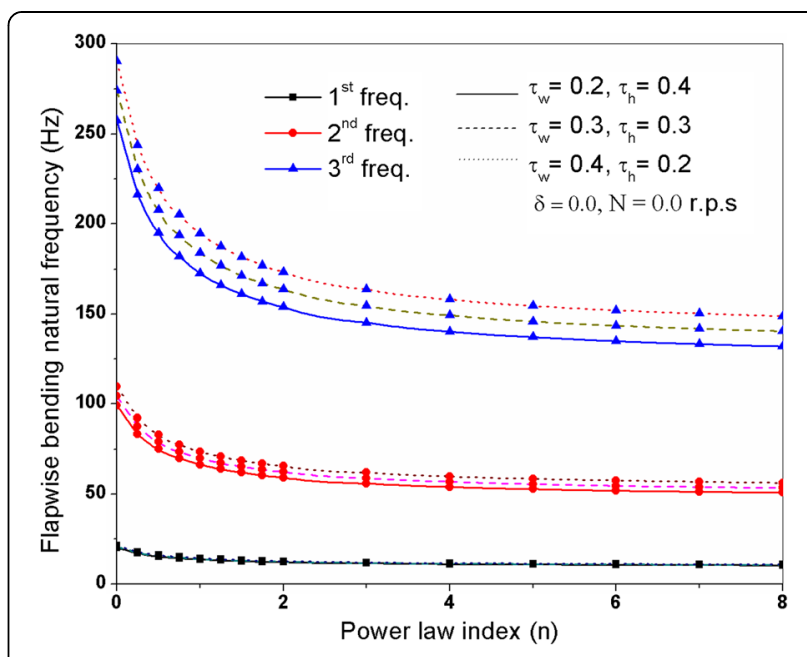

Fig. 2 Effect of taper ratio and power law index on flapwise bending natural frequencies

$$
K_{i j}^{B a}=\int_{0}^{1}\left(1-\tau_{b} \xi\right)^{3}\left(1-\tau_{h} \xi\right) \psi_{a i}^{\prime \prime} \psi_{b j}^{\prime \prime} d \xi
$$

and

$$
\begin{aligned}
K_{i j}^{G a} & =\int_{0}^{1} \delta\left(1-\tau_{w} \xi\right)\left(1-\tau_{h} \xi\right)(1-\zeta) \psi_{a i}^{\prime} \psi_{b j}^{\prime} d \zeta \\
& +\frac{1}{2} \int_{0}^{1}\left(1-\tau_{w} \xi\right)\left(1-\tau_{h} \xi\right)\left(1-\zeta^{2}\right) \psi_{a i}^{\prime} \psi_{b j}^{\prime} d \zeta
\end{aligned}
$$

where $\psi_{a i}$ is a function of $\xi$ that has the same functional value of $x$.

From Eq. 30, an eigenvalue problem can be derived by assuming that $\theta s$ are harmonic functions of $\tau$ expressed as

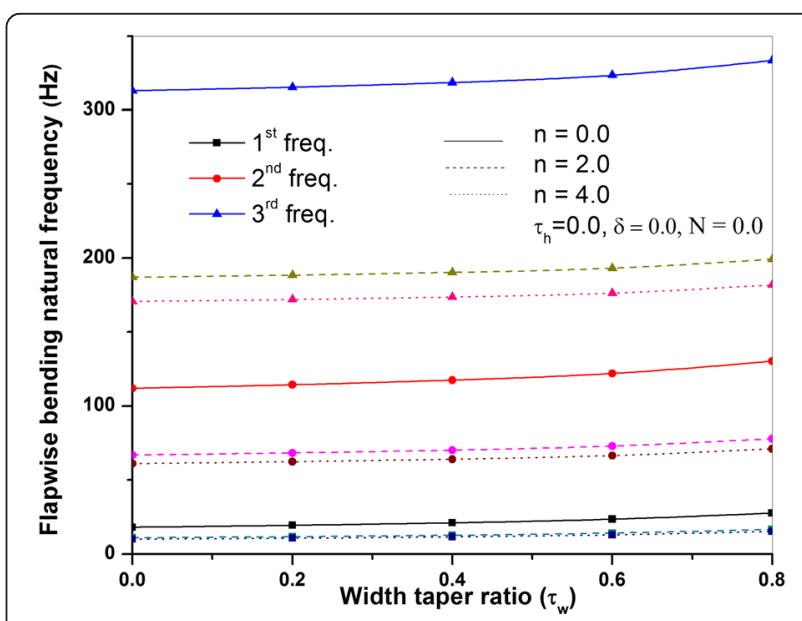

Fig. 3 Effect power law index on lowest three flapwise bending natural frequencies versus width taper ratio 


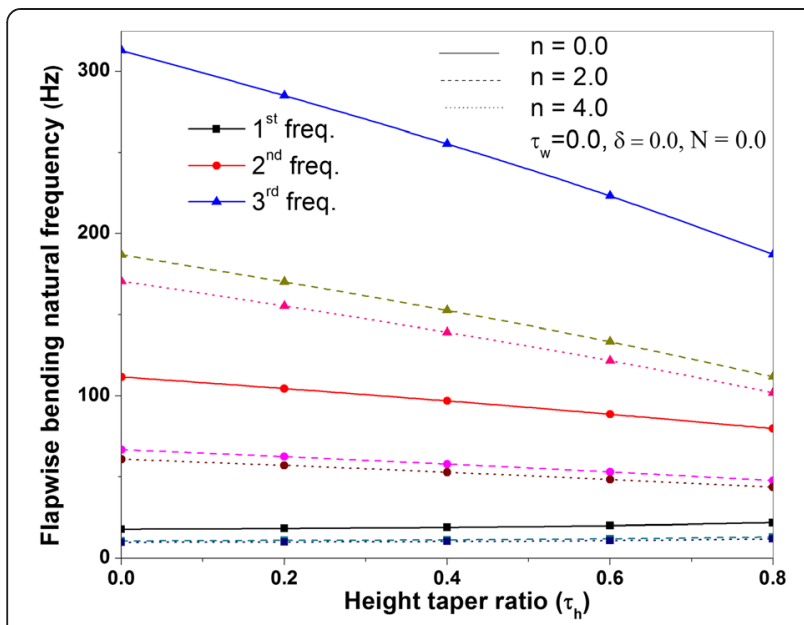

Fig. 4 Effect of power law index on lowest three flapwise bending natural frequencies versus height taper ratio

$$
\theta=e^{j \omega \tau} \Theta
$$

where $j$ is the imaginary number, $\omega$ is the ratio of the flapwise bending natural frequency to the reference frequency, and $\Theta$ is a constant column matrix characterizing the deflection shape for synchronous motion and this yields

$$
\omega^{2} M \Theta=K^{C} \Theta
$$

where $M$ is mass matrix and $K C$ is stiffness matrix, whose elements are defined as

$$
\begin{aligned}
M_{i j} & =M_{i j}^{33} ; \\
K_{i j}^{C} & =K_{i j}^{B 3}+\gamma^{2}\left(K_{i j}^{G 3}-M_{i j}^{33}\right)
\end{aligned}
$$

\section{Results and discussion}

The procedure presented above for free vibration analysis of a double-tapered beam is demonstrated through numerical examples.
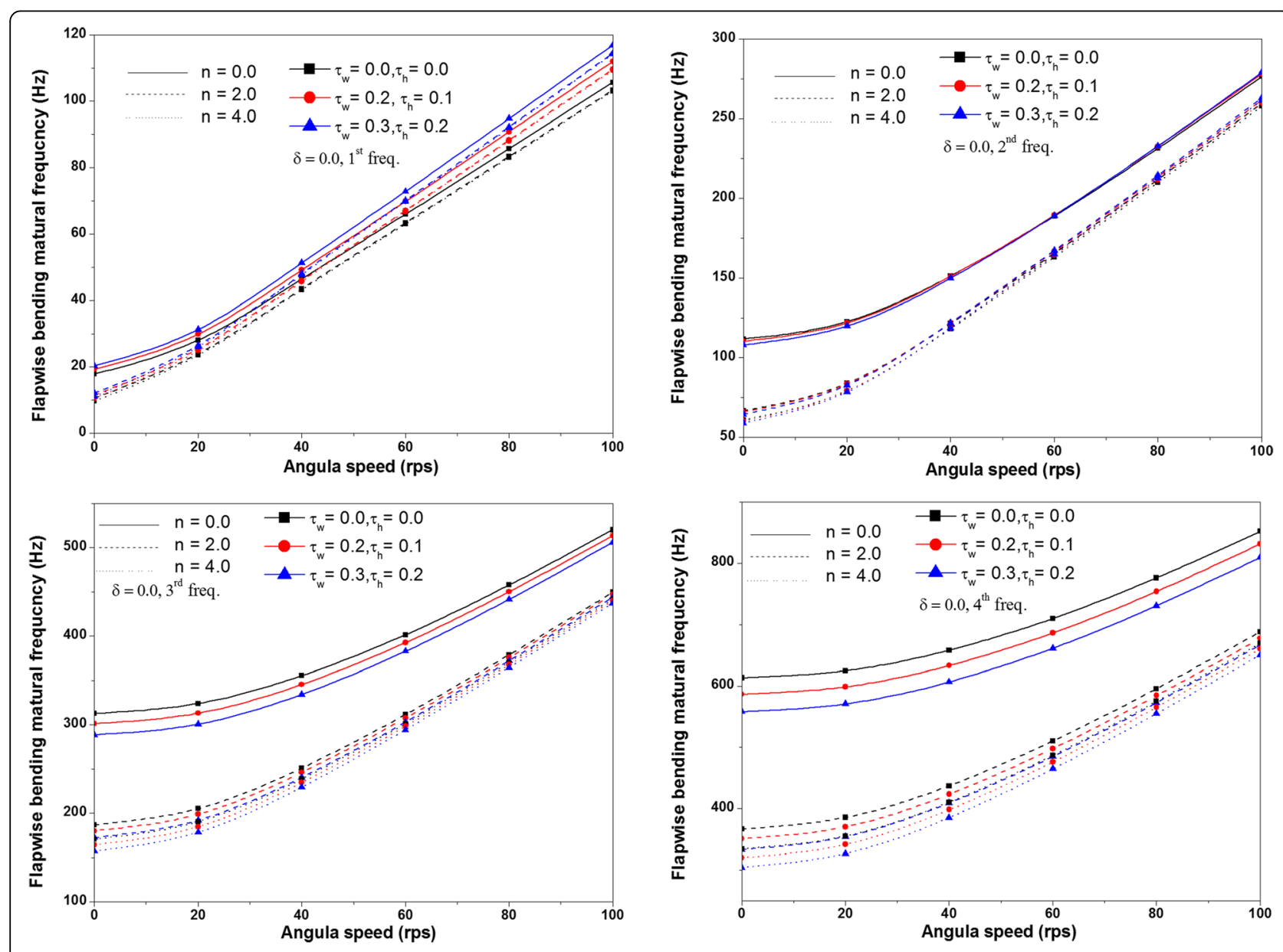

Fig. 5 Effect of taper ratio and power law index on flapwise bending natural frequencies versus angular speed $\left(\tau_{w}>\tau_{h}\right)$ 
The results from the present modeling method have been obtained considering ten assumed modes compared with those reported by Ozgumus and Kaya (2006) using the differential transform method. The influence of taper ratios on the first three fundamental frequencies is presented in Tables 1 and 2 and the results are compared.

From the data, it has been observed that there is nearly one-to-one correspondence between the results obtained from the current approach and those reported based on the differential transform method.

In view of this observation, it is opined that the current approach is in order and has been adopted for further analysis in this study.

Further analysis has been carried out on double-tapered functionally graded beam made of steel as metallic constituent and alumina as ceramic constituent whose mechanical properties are given in Table 3 . The dimensional parameters length $=1000 \mathrm{~mm}$, breadth $=20 \mathrm{~mm}$, and height $=10 \mathrm{~mm}$ are considered for the analysis.

The frequencies are evaluated with ten assumed modes to obtain the converged bending natural frequencies. As the frequencies obtained with number of modes greater than ten did not show any significant improvement in the results, the number of assumed modes is restricted to ten only.

The effect of power law index and taper ratios on flapwise bending natural frequencies of a stationary functionally graded double-tapered beam is presented in Fig. 2. It is observed that the three frequencies decrease with an increase in power law index, $n$, and when the beam composition approached a more metallic state, the rate of decrease in these frequencies is predominant up to a certain $n$ value, thereafter the frequencies are relatively un-effected by increase in $n$ value at all taper ratios. The variation in flapwise bending natural frequencies with the increase in taper ratios is decreasing at all power law indices in all modes of vibration, as the change in taper ratio alters the stiffness of the beam.

The effect of taper and power law index on frequencies of a single-tapered beam is studied. The variation of flapwise bending natural frequencies of the functionally graded rotating stationary beam with respect to width and height taper ratios has been presented in Figs. 3 and 4.
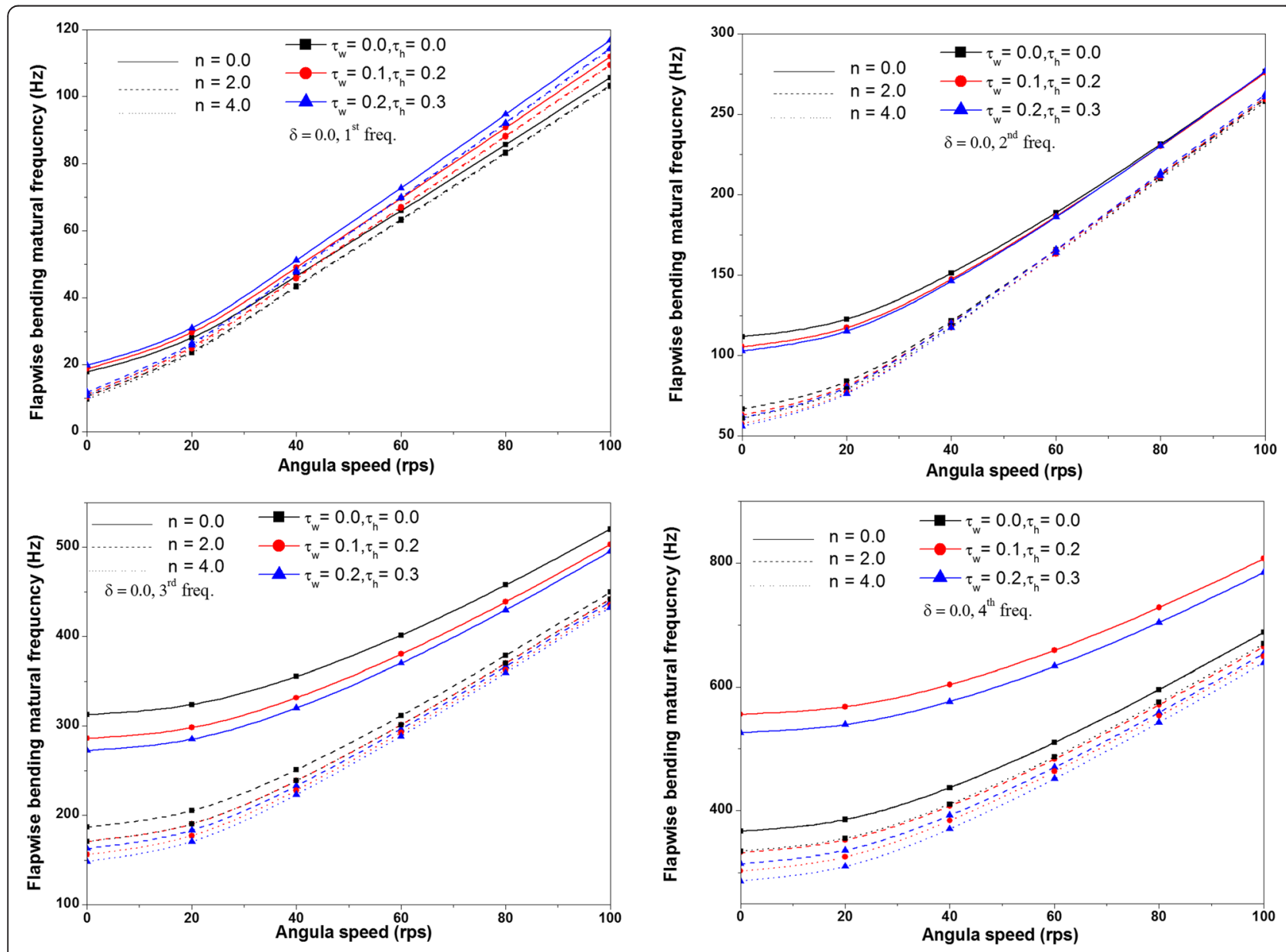

Fig. 6 Effect of taper ratio and power law index on flapwise bending natural frequencies versus angular speed $\left(\tau_{W}<\tau_{h}\right)$ 
For all the values of power law index, all frequencies are observed to increase with the increase in height taper ratio and the first flapwise frequency is observed to increase while a decreasing trend has been observed in case of higher frequencies with an increase in width taper ratio. It is also observed that, at higher values of power law index, the variation of flapwise bending natural frequencies with a variation in width and height taper ratios tends to reduce.

The influence of angular speed on flapwise bending natural frequency for different taper ratios and power law indices is presented in Figs. 5 and 6. It has been observed that the first frequency has a lower magnitude for uniform beam compared to that for tapered beam and a reverse trend has been observed in respect of higher order frequencies for all the taper ratios of the beam. It is further observed that the variation in frequencies is predominant when $\tau_{w}<\tau_{h}$ as compared to the case of $\tau_{w}>\tau_{h}$ though the area of beam cross section is the same in both cases.
It has been observed that for different values of taper ratios and power law indices, the fundamental frequencies are diverging with the increase in angular speed and all higher order frequencies are observed to be converging. However, at a given speed, the frequencies decrease with an increase in power law index.

It is also observed that the influence of taper ratio on fundamental flapwise bending natural frequency is increasing with the increase in angular speed and the influence is less on higher modes of vibration. The power law index has negligible influence on the trend of flapwise bending natural frequencies with the increase in angular speed for different taper ratios which is evident from similarity of variation pattern for solid $(n=0)$ and that for dashed $(n=2.0)$ and dotted $(n=4.0)$ lines for all modes of vibration. These trends should be expected that the tapered beams under consideration would behave differently, as the stiffness at the root and load variation along the length of the beam depend on the taper ratio.
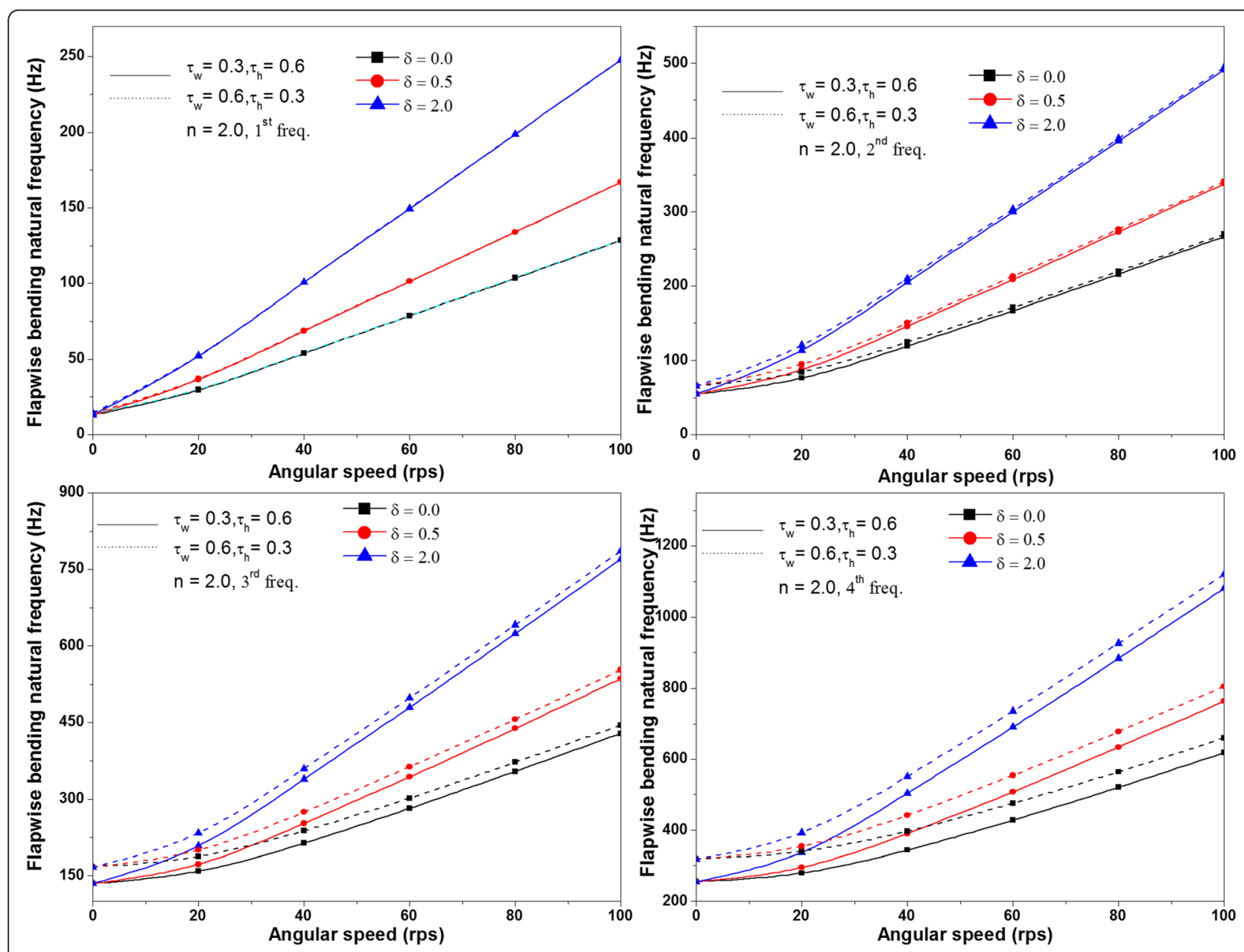

Fig. 7 Effect of hub radius ratio and taper ratio on flapwise bending natural frequency versus angular speed 
The effect of hub radius ratio and beam taper ratio on flapwise bending natural frequencies of the rotating tapered beam is presented in Fig. 7. In general, the flapwise bending natural frequencies increase with the increase in angular speed. From the figure, it is observed that the difference in frequencies for the two geometries of the beam is more at lower speeds and is decreasing as the speed increases. It may be also noted that the rate of increase in frequencies increase with an increase in hub radius ratio and the increase in ratio of $\tau_{b} / \tau_{h}$. The hub radius ratio could not influence the rate of increase of flapwise bending natural frequency with the increase of angular speed. This is evident from uniformity in the gap between the solid line and dashed lines. These trends should be expected from the increase in angular speed, and hub radius leads to stiffening effect of rotation and increase in taper ratio results to softening effect resulting from the decrease of the cross-sectional area.

\section{Conclusions}

The free flapwise bending vibrations of a rotating tapered beam are investigated in this work. The equations of motions are derived using hybrid deformation variables employing Lagrange's approach. Rayleigh-Ritz method is used to evaluate the frequencies of the beam. Linear tapering in breadth and depth directions is considered for the analysis. Variation in taper ratios affects the frequency parameters of the beam. The frequency parameters are observed to increase with the increase in hub radius parameter. The power law index has negligible influence on the trend of flapwise bending natural frequencies with the increase in angular speed for different taper ratios.

\section{Competing interests}

The authors declare that they have no competing interests.

\section{Authors' contributions}

NVRM carried out the analytical solution and MRN conceived the study and participated in concept checking and MS preparation. Both the authors read and approved the final MS.

\section{Author details}

${ }^{1}$ Department of Mechanical Engineering, Nalla Malla Reddy Engineering College, Hyderabad, Telangana 500088, India. ${ }^{2}$ Department of Mechanical Engineering, JNTUK College of Engineering Vizianagaram, Vizianagaram, Andhra Pradesh 535003, India.

Received: 24 March 2015 Accepted: 29 June 2015

Published online: 14 August 2015

\section{References}

Aminbaghai, M, Murín, J, \& Kutiš, V. (2012). Modal analysis of the FGM-beams with continuous transversal symmetric and longitudinal variation of material properties with effect of large axial force. Eng Struct, 34, 314-329.

Babu Gunda, J, \& Ganguli, R. (2008). New rational interpolation functions for finite element analysis of rotating beams. Int J Mech Sci, 50(3), 578-588.

Banerjee, JR, \& Jackson, DR. (2013). Free vibration of a rotating tapered Rayleigh beam: a dynamic stiffness method of solution. Comput Struct, 124, 11-20.

Cheng, Y, Yu, Z, Wu, X, \& Yuan, Y. (2011). Vibration analysis of a cracked rotating tapered beam using the $p$-version finite element method. Finite Elem Anal Des, 47(7), 825-834.
Das, D, Sahoo, P, \& Saha, K. (2009). Out-of-plane free vibration analysis of rotating tapered beams in post-elastic regime. Mater Des, 30(8), 2875-2894.

Ganesh, R, \& Ganguli, R. (2013). Stiff string approximations in Rayleigh-Ritz method for rotating beams. Appl Math Comput, 219(17), 9282-9295.

Gunda, JB, Gupta, RK, \& Ganguli, R. (2009). Hybrid stiff-string-polynomial basis functions for vibration analysis of high speed rotating beams. Comput Struct, 87(3-4), 254-265.

Hosseini, SAA, \& Khadem, SE. (2007). Vibration and reliability of a rotating beam with random properties under random excitation. Int J Mech Sci, 49(12), 1377-1388.

Huang, CL, Lin, WY, \& Hsiao, KM. (2010). Free vibration analysis of rotating Euler beams at high angular velocity. Comput Struct, 88(17-18), 991-1001.

Kadoli, R, Akhtar, K, \& Ganesan, N. (2008). Static analysis of functionally graded beams using higher order shear deformation theory. Appl Math Model, 32(12), 2509-2525.

Lin, S-M, Lee, J-F, Lee, S-Y, \& Wang, W-R. (2006). Prediction of vibration of rotating damped beams with arbitrary pretwist. Int J Mech Sci, 48(12), 1494-1504.

Murin, J, Aminbaghai, M, Hrabovský, J, Kutiš, V, \& Kugler, S. (2013). Modal analysis of the FGM beams with effect of the shear correction function. Compos Part B, 45(1), 1575-1582.

Ozgumus, O, \& Kaya, M. (2006). Flapwise bending vibration analysis of double tapered rotating Euler-Bernoulli beam by using the differential transform method. Meccanica, 41(6), 661-670

Sankar, BV. (2001). An elasticity solution for functionally graded beams. Compos Sci Technol, 61(5), 689-696.

Tsai, MH, Lin, WY, Zhou, YC, \& Hsiao, KM. (2011). Investigation on steady state deformation and free vibration of a rotating inclined Euler beam. Int J Mech Sci, 53(12), 1050-1068.

Ying, J, Lü, CF, \& Chen, WQ. (2008). Two-dimensional elasticity solutions for functionally graded beams resting on elastic foundations. Compos Struct, 84(3), 209-219.

Yoo, HH, \& Shin, SH. (1998). Vibration analysis of rotating cantilever beams. J Sound Vib, 212(5), 807-828.

Ziane, N, Meftah, SA, Belhadj, HA, Tounsi, A, \& Bedia, EAA. (2013). Free vibration analysis of thin and thick-walled FGM box beams. Int J Mech Sci, 66, 273-282.

\section{Submit your manuscript to a SpringerOpen ${ }^{\odot}$ journal and benefit from:}

- Convenient online submission

Rigorous peer review

- Immediate publication on acceptance

- Open access: articles freely available online

- High visibility within the field

- Retaining the copyright to your article

Submit your next manuscript at springeropen.com 